\title{
A MIXED RADIOPAQUE - RADIOLUCENT LESION IN THE ANTERIOR MANDIBLE ASSOCIATED WITH MULTIPLE IMPACTED TEETH - A RADIODIAGNOSTIC CHALLENGE?
}

Bhandarkar Gowri Pandarinath ${ }^{1}$, Shetty Kushal ${ }^{2}$

1) Department of Oral Medicine and Radiology, A. J. Institute of Dental Sciences, Mangalore, India.

2) Department of Pedodontic, A. J. Institute of Dental Sciences, Mangalore, India.

\section{ARTICLE INFORMATION:}

Article History:

Received: 14 February, 2017

Accepted in revised form: 27 June, 2017

Published:1 August, 2017

Corresponding author:

Bhandarkar Gowri Pandarinath

E-mail: id-gowribhandarkar@gmail.com

Keyword:

Desmoplastic Ameloblastoma, mandible, mixed radiopaque-radiolucent lesion, multiple impacted teeth, odontogenic tumor, stromal desmoplasia.

\section{ABSTRACT:}

Desmoplastic ameloblastoma (DA) is an unusual variant of ameloblastoma characterized by abundant dense collagenous stroma (desmoplasia) with small nests and strands of an odontogenic epithelium. The stroma dominates over the epithelial neoplastic component. The case report of Desmoplastic variant we are presenting is unique and noteworthy in that it exhibited unusual radiographic features; a mixed radiopaque-radiolucent lesion that resembled a benign fibro-osseous lesion. It was seen in the anterior mandible and it was also associated with multiple impacted teeth which is quite a rarity.

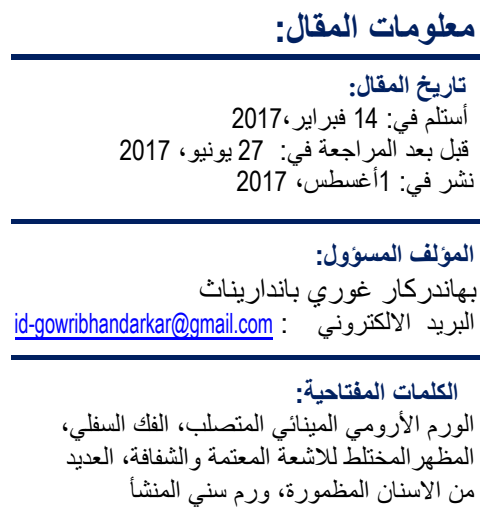

الملخص العربي

المظهر المختلط للاشعة المعتمة والثفافة في مقدمة القك السفلي والمرتبطة

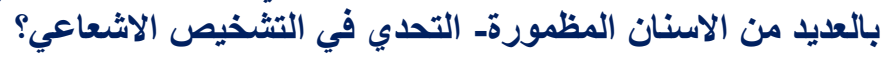

بهاندركار غوري بانداريناث1، شيتي كوشال2

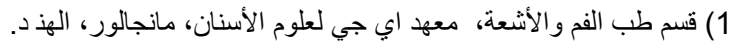

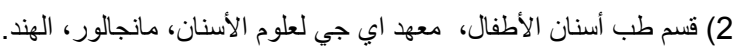

الورم الأرومي المينائي المتصلب (DA) هو متغير غير عادي من الورم الأرومي المينائي الذي

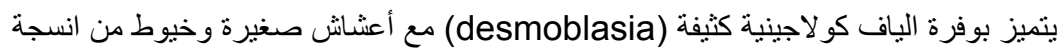
ايييثلية سنية. التي تسيطر على عنصر الورم الظهاري. تقرير حالة مختلفة من الورم الأرومي التي اعني

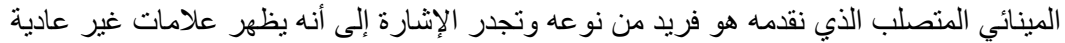

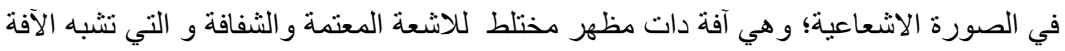
الليفية العظمية الحميدة. تم رؤيتها في مقدمة الفك السفلي و المرتبطة بالعديد من الاسنان المظمورة و التي تعتبر من الحالات النادرة.

Copyright $\odot$ 2017. LDJ. This is an open access article distributed under the Creative Commons Attribution 3.0 License, which permits unrestricted use, distribution, and reproduction in any medium, provided the original work is properly cited.

Citation:Libyan Dent J 2017, 7: 25320831 -http://dx.doi.org/10.5542/LDJ.v7i0.25320831 


\section{INTRODUCTION}

Ameloblastoma is the most common benign odontogenic tumor of epithelial tissue origin. Desmoplastic Ameloblastoma (DA) is a variant. Eversole et al. ${ }^{1}$ gave the first detailed report on the desmoplastic variant of ameloblastoma in the English literature in 1984. He described 3 cases and called it 'ameloblastoma with pronounced desmoplasia'.

The growing knowledge regarding the clinical, radiographic presentation and pathology of DAs has led to inclusion as a distinct variant of ameloblastoma in the World Health Organization (WHO) classification of odontogenic tumors. ${ }^{2}$

This report attempts to help the clinicians in developing familiarity with the clinical presentation of desmoplastic ameloblastoma and recognize such cases. The intention of this article is to report a case of desmoplastic ameloblastoma with the characteristic clinical, radiographic and histological features and to contribute to the dentists a better understanding of this variation in an odontogenic tumor.

\section{CASE REPORT}

A 35-year-old female reported to our department with a swelling in the lower anterior region of the jaw since six months (Fig 1).

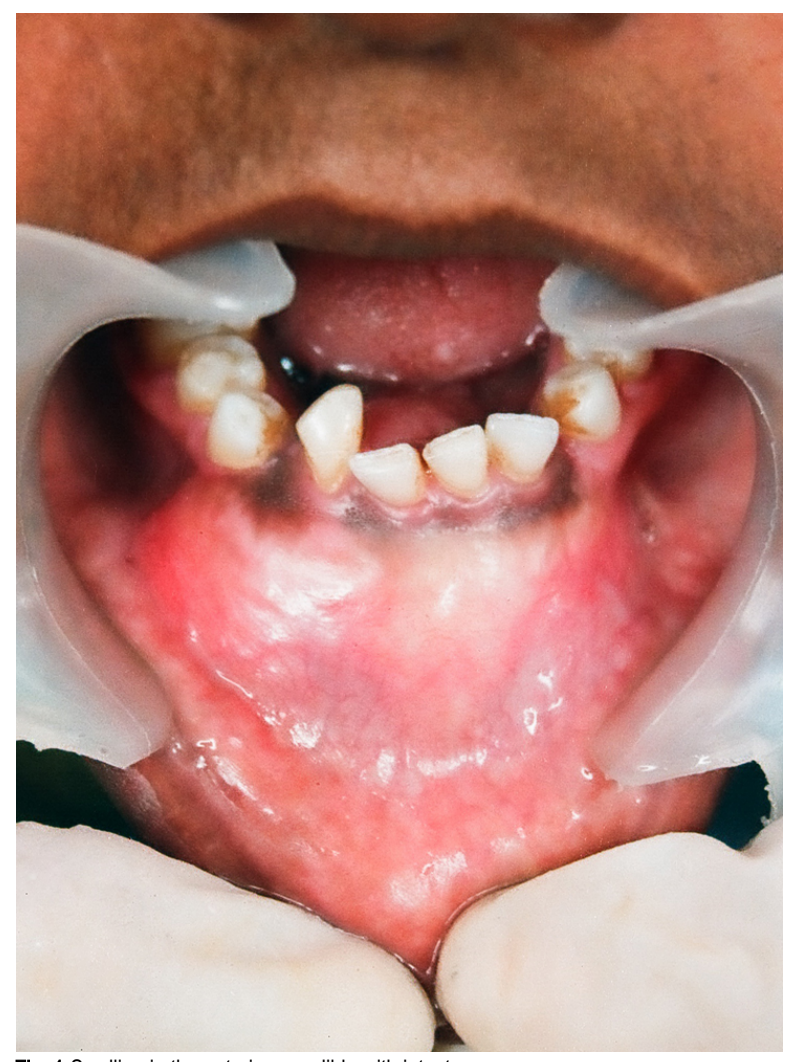

Fig. 1:Swelling in the anterior mandible with intact mucosa.
History dates back to six months when she noticed a small swelling that spontaneously increased to the present size which was associated with mobility of lower anterior teeth and without history of bleeding or any sensory changes. There was no history of trauma and the past medical and dental history were unremarkable and non-contributory. On general examination, no lymphadenopathy, fistulae or sinus openings were present, and examination of TMJ revealed no abnormality. Intra-oral examination revealed missing 23,33 and 43 without any history of extraction.

Local, extra-oral clinical examination revealed a well-defined solitary swelling in the lower one-third of the face in the region of the symphysis that was oval in shape measuring about $6 \times 4 \mathrm{~cm}$ in size. It was extending from the lower lip to the inferior border of the mandible, and it was extending from left parasymphyseal area crossing the midline to involve the right parasymphyseal area. The skin over the swelling appeared smooth and borders were well defined with obliteration of the mentolabial fold. It was non-tender on palpation. The swelling was bony hard in consistency with no signs of paraesthesia. Intra-orally, the swelling measured $5 \times 3 \mathrm{~cm}$ extending from right second premolar to left first premolar of the mandible with marked obliteration of the labial vestibule. Expansion of both labial and lingual cortical plates of the mandible were evident. The mucosa over the swelling was smooth, intact and of normal mucosal color. The examination also revealed missing 33 and 43 . On palpation, the swelling was tender in the region of the right second premolar. The consistency of the swelling was bony hard. $31,32,41$ and 42 were of grade 2 mobility. 31 and 41 showed a negative response to vitality test on electric pulp testing. A provisional diagnosis in favor of an adenomatoid odontogenic tumor was given based on history and clinical findings. Desmoplastic ameloblastoma and fibro-osseous lesion were given as differential diagnoses.

Mandibular occlusal radiograph of the lesion revealed the presence of a single, ill-defined, mixed radiopaque-radiolucent lesion (Fig 2). It was extending from the mesial aspect of 34 to the distal aspect of 45 with the marked expansion of the labial cortical plate and mild expansion of the lingual cortical plate. There were small flecks of radioopacities seen against radiolucent background 


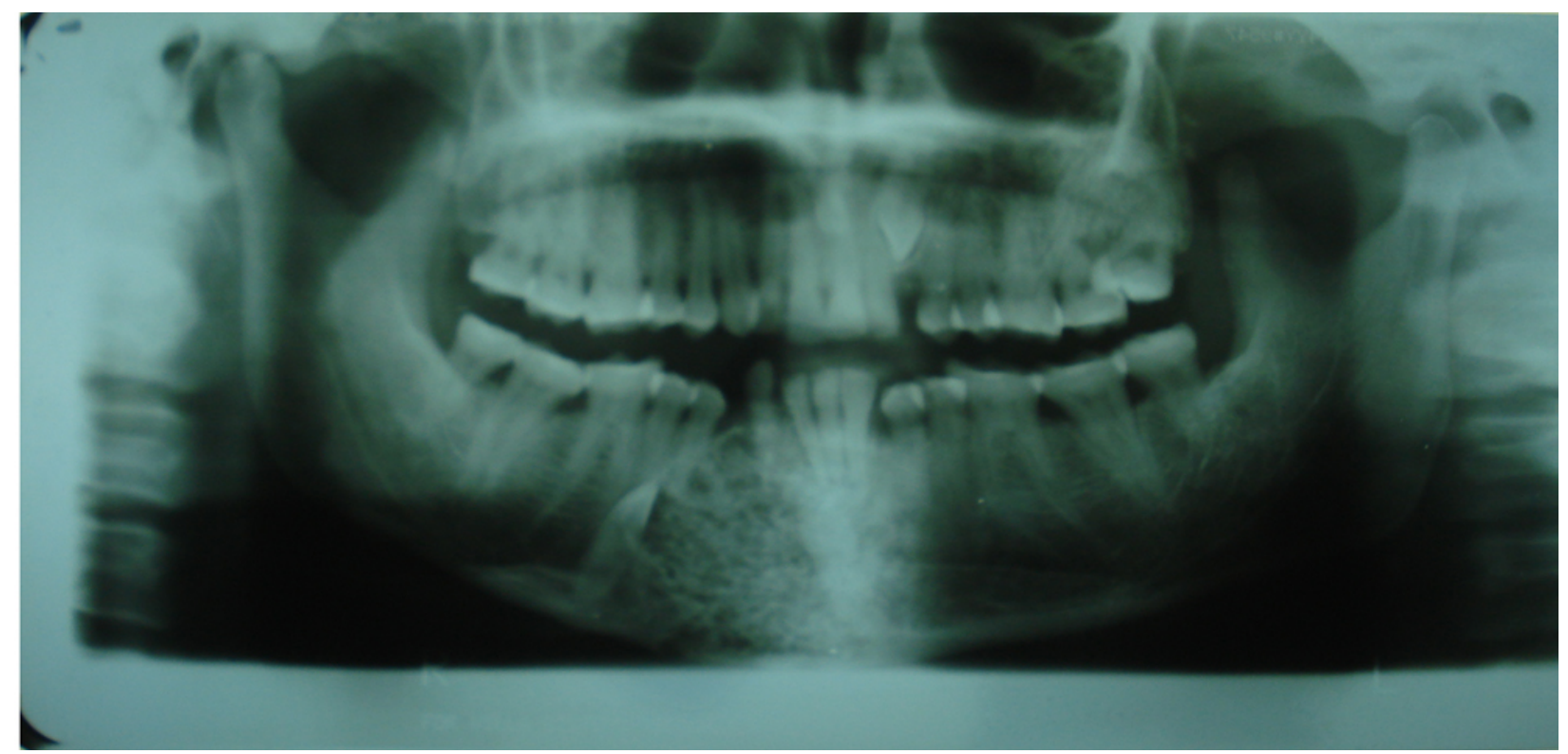

Fig 2: Panoramic radiograph showing mixed radiolucent radiopaque lesion with impacted 33,43 . Also showing impacted 23 and mesiodens.

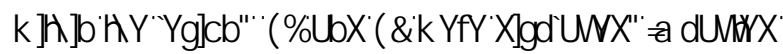

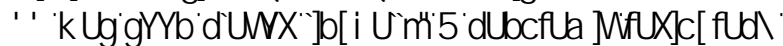
UHMDOGG D] FRP SOHAL DEMHCFH R『 QRLP DOWDEHFXOH

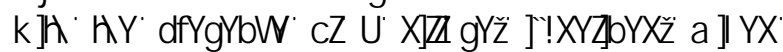

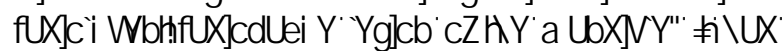

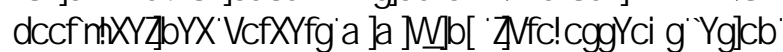

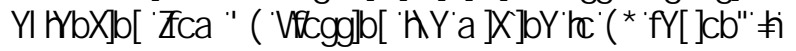

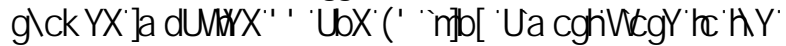
LQHURUERLCHURIIKHHP DQGEOH LQ WKHI Y P SKI VHDODQG SDIDV PSKI VHDO UHJIRQ UHSHFUYHOII 7KH OMRQ

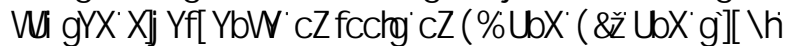

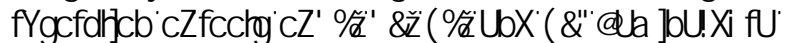

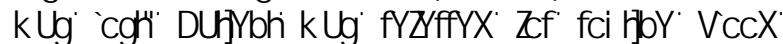

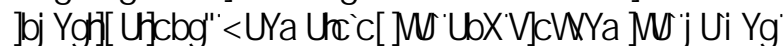
ZHHZ LKLQQRIP DODQJH

: UKX DQ LQRUP HG FRQMHQW DQ LQFURQDOEIRSV $\square$ ZDV SHURLP HGI DQG WXH KLWRSDUKRQPJIF UHSRUWMRZHG

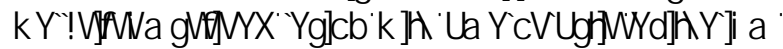

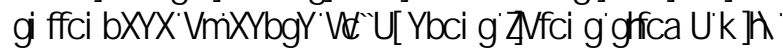

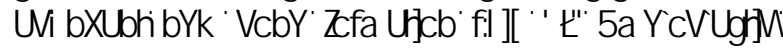
IOOQV DWSOFHM WKRZHG F WF FKDQJHM ) HDXUM

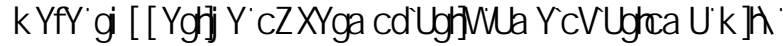
QHZIERQHIRIP DURQ

7KH WXPRU KDG EHQ RSHOHG DQG WKH DQAMRU P DQGEOIL HMFFAG DQG UFRQWWKFAG XVQJ DU UE] JUDIW

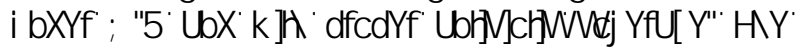
JURW VXUIFDO VSHFP HQ KDG EHQQ JLHQ IRU WXH KLYRSDUKRQEJIFDO UHSRUW ZKIFK FDPH DV WKH GHP RSOWFI YDUDQMRI DP HOREOWRP DI 7KH SDUHQNV SRMASHOUNH SHURG ZDV XQYMHQNKO DQG

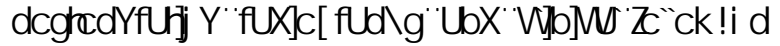

H DP LQDARQ RI WXH SDMFQMRUUR I HDW GVFGMHG QR HMGHQFH RI UFFXUHCFH RU UHMGXDO WXP RU \$Q WXH

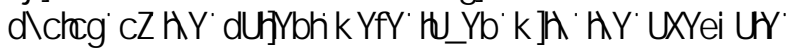

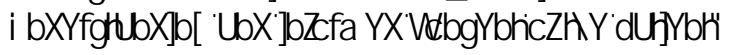

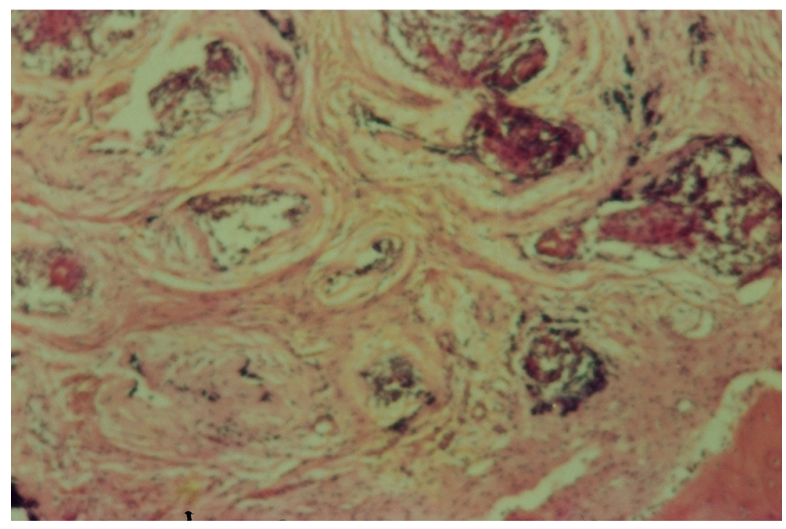

Fig 3: Showing ameloblastic epithelium surrounded by dense collagenous fibrous stroma with abundant new bone formation. (low power).

\section{DISCUSSION}

In 1984, Eversole et al. ${ }^{1}$ described a desmoplastic variant of ameloblastoma(DA). DA exhibits important differences in gender, anatomic distribution, radiographic findings and histologic appearance compared with other types of ameloblastoma. The incidence of DA is relatively low ranging from $0.9 \%$ $-12.1 \%$ of all the ameloblastomas. ${ }^{3}$

Kaffe et al. ${ }^{4}$ reported following findings; The mean age of the patients with this variant is 42.3 years (range 17-70 years). The desmoplastic variant was found to be more common in females (as was in our case), the 
male-female ratio was 1:2. Data from different geographical regions seem to suggest a biogeographical pattern in that the relative frequency of desmoplastic ameloblastoma is slightly higher in an Asian population. Regarding its origin, it is suggested that DA develops from the periodontal membrane of the related tooth and that it might arise from epithelial rests of Malassez in the periodontal membrane. In this case, the disappearance of lamina dura was seen w.r.t 31, 32, 41 and 42.

The desmoplastic ameloblastoma appears to be unique; approximately half of the desmoplastic lesions has been located in the maxilla. The vast majority of them occur in the anterior or premolar portion of the jaws. ${ }^{3}$ In our case, DA was seen in the anterior mandible. Higuchi et al. postulated that ameloblastoma in the tooth bearing area had a greater tendency to have an abundant stroma and to be desmoplastic. Genetic or biologic differences of the bone is due to the anatomical sites such as alveolar bone proper of odontogenic origin versus body of the jaw bone of non-odontogenic origin. It might be attributed to the histopathological variation of ameloblastomas. $^{3}$

The radiographic appearance of this neoplasm is that of a mixed radiolucent/radiopaque lesion which suggests that the tumor may be more aggressive than other variants of ameloblastoma. It is also believed that the mixed pattern expresses the infiltrative nature of the tumor. The infiltrative behavior of the tumor attributes to the ill-defined borders. There is involvement of lamina-dura. Radiologically, DA is seen either as ill-defined mass containing osteolytic and sclerotic areas. It can also occur as multifocal radiodense flecks within radiolucent background resembling a honeycomb. This is because of the infiltration of tumor cells into the adjacent marrow spaces with simultaneous vigorous osteoblastic activity. ${ }^{2}$ Tooth displacement was a common feature of this variant ( $92 \%$ of the cases) as was seen in our case also. Displacement of the adjacent teeth 42 and 41 was seen and also tumor had been associated with missing teeth, 33 and 43 . Root resorption had been discovered in only $33 \%$ of the cases. In our case also slight root resorption was seen with respect to 31,32 , 41 and 42.

The radiographic differential diagnosis include fibroosseous lesions like cemento-ossifying fibroma(if well circumscribed), cementoma (intermediate stage), osteitis, cementoblastoma, fibrous dysplasia, calcifying odontogenic cyst, chronic sclerosing osteomyelitis.
Thus, desmoplastic ameloblastoma should primarily be included in the differential diagnosis of a mixed radiopaque-radiolucent lesion with diffuse borders in the anterior premolar region of the jaws. ${ }^{5}$ Islands of ameloblastic appearing columnar cells that surround spindle-shaped cells that resemble stellate reticulum and a stroma with marked desmoplasia $^{3}$ characterize the histological pattern.

It is speculated that the desmoplasia in DA might act as a limiting barrier for the local spread of the DA tumor cells compared with the situation in conventional ameloblastoma where such barrier is absent. ${ }^{6}$ Histologically the DA cases were grouped into the following two types: 1) simple desmoplastic type which is predominant $(88.0 \%)$; and 2) desmoplastic with osteoplasia type which is rare $(12.0 \%)$. The simple desmoplastic type showed histologic features of extensive desmoplasia of the stroma, whereas the desmoplastic with osteoplasia type showed histologic characteristics that exhibit features of the simple desmoplastic type and additional calcific structures. ${ }^{6}$ This explains the radiologic appearance of mixed radiolucency and radio-opacity thereby presenting radiographic features of a fibro-osseous lesion. ${ }^{9}$ Our case is an example of DA with osteoplasia variant which is a rare type.

Philipsen et al. ${ }^{7}$ remarked that DA has a tendency to produce de novo synthesis of extracellular fibrous protein, which has been attributed to desmoplasia seen in this tumor. Furthermore, the de novo synthesized extracellular protein could serve as nidus for calcification seen in the DA with osteoplasia.

Histological differential diagnosis includes odontogenic fibroma, squamous odontogenic tumor, ameloblastic fibroma, squamous cell carcinoma. The characteristic palisading layer of ameloblastoma may not be seen in all the epithelial clusters, especially if the biopsy specimen is small. Areas with only narrow strands of epithelial cells within desmoplastic stroma may appear as odontogenic fibroma; but the clinical behavior and management of these two tumors is different. Ameloblastoma is a potentially aggressive tumor that needs an en bloc resection. The Odontogenic fibroma is much less aggressive, and enucleation is probably curative. $^{8}$

Another differential diagnosis is the squamous odontogenic tumor that may have a fibrotic stroma. The squamous metaplasia observed in some areas of 
the desmoplastic variant of ameloblastoma may mimic it if the palisading layer of the tall columnar cells has not been identified. Although some cases of the squamous odontogenic tumor may have an aggressive clinical course, the currently preferred treatment is curettage, which is followed by few recurrences. Other differential diagnosis includes squamous cell carcinoma and ameloblastic fibroma. The former has prominent cytological atypia while the latter has a cellular stroma. Accurate diagnosis of the desmoplastic variant of ameloblastoma depends on identification of the typical ameloblastic areas. This may require examination of more tissue or a repeated biopsy. ${ }^{8}$

Compared with the SMAs, $\mathrm{Ng}$ and Siar using various immunohistochemical studies have identified DA tumor cells as showing variable expression of S-100 protein and desmin. There was also a high expression of caspase- 3 and Fas, decreased expression of CK19 and high expression of $p 63 .{ }^{9}$ The desmoplastic stroma of DA has been reported to show a strong positive reaction to collagen type $\mathrm{VI}^{7}{ }^{7}$ This has been interpreted as an active de novo synthesis of extracellular matrix protein hence ruling out the scar tissue, immunonegativity for tenascin and strong immunopositive reaction for fibronectin and type I collagen. ${ }^{9}$ There was also marked immunoexpression of TGF- $\beta$. In one study, Immunolocalization of transforming growth factor beta (TGF-B), one of most potent local factors for modulating extracellular matrix formation was observed in DA. This had been done in order to study its participation in the stromal desmoplasia. These results show that TGF- $\beta$ produced by tumor cells of DA play a role in the desmoplastic matrix formation. ${ }^{9}$ In contrast to ordinary ameloblastomas, marked immunoexpression was observed in all DAs but one. ${ }^{9}$

With limited information of its biologic behavior and prognosis, resection is the most sought after option for desmoplastic ameloblastoma although some cases have been treated by enucleation and curettage. The desmoplastic variant has a potential for recurrence $(21.4 \%)$ because it fails to form a connective tissue capsule. $^{4}$

The exact interface of the lesion with normal bone, makes it especially difficult to be treated surgically. ${ }^{10}$ Desmoplastic ameloblastomas tend to infiltrate between bone trabeculae and curettage often leaves islands of the tumor within the bone, which later manifest as recurrences. ${ }^{10}$ It needs to be investigated whether the recurrence is due to the incomplete surgery.
Therefore, block excision is the most widely used treatment to avoid recurrence. ${ }^{10}$ In our case also, wide resection of the anterior mandible was done and reconstructed using a rib: graft under G.A and with proper antibiotic coverage.

The authors feel that until proven otherwise, the desmoplastic variant must be managed with surgical resection. In addition, close longterm follow-up is indicated in these patients. What makes our case interesting and novel from past reports is that DA in our case involved the anterior mandible, it had been associated with two impacted teeth 33 and 43 and histologically it had been diagnosed as DA with osteoplasia variant which was quite rare. How the finding advances knowledge of the field is that the report of this case adds to the literature of mixed radiolucent-radiopaque lesions which may not always be histopathologically diagnosed as a fibro-osseous lesion but could turn out to be a desmoplastic ameloblastoma. It is said "A forewarned is a forearmed" This report also benefits the dental community by cautioning them to be aware of a mixed radiolucentradiopaque lesion like DA which can be associated with multiple impacted teeth which is quite a rare finding.

\section{CONCLUSION}

In view of the rarity of desmoplastic ameloblastoma case series, it continues to be one of the nature's secrets. Specific clinical, imaging and histological features characterizes the desmoplastic ameloblastoma. For proper understanding of such cases, more profound scrutiny and long-term follow-up is required. The clinician has to be alert regarding the unusual presentation of this neoplasm. The definite diagnosis always follows histopathological examination. The lesion will remain a mystery and it is prudent that a more definite tumor analysis techniques must be pursued with aggressive follow-up and as well track as many more cases into medical literature as possible. 


\section{REFERENCES}

1. Eversole LR, Leider AS, Hansen LS. Ameloblastomas with pronounced desmoplasia. J.Oral Maxillofac. Surg 1984;42:734-740.

2. Kishino M, Mmakami S, Fukada Y, Ishida T. Pathology of the desmoplastic ameloblastoma. J Oral Pathol Med 2001;30:35-40.

3. Higuchi $\mathrm{Y}$, Nakamura $\mathrm{N}$, Ohishi M, Tashiro $\mathrm{H}$. Unusual ameloblastoma with extensive stromal desmoplasia. J of craniomaxillofac surg 1991;19:323-327.

4. Kaffe I, Buchner A, Taicher S. Radiological features of desmoplastic variant of ameloblastoma. Oral surg Oral med Oral pathol 1993;76:525-529.

5. Rastogi $R$, Jain $H$. Desmoplastic ameloblastoma: Case report. Indian J Radiol Imaging 2008;18:53-55.

6. Effiom OA, Odukoya O. Desmoplastic ameloblastoma: analysis of 17 Nigerian cases. Oral Surg Oral Med Oral Pathol Oral Radiol Endo 2011;111: e27-e31.

7. Philipsen HP, Ormiston IW, Reichart PA. The Desmo and osteoplastic ameloblastoma. Histological variant or clinico-pathological entity? Case report. Int. J Oral maxillofac surg 1992;21:352-357.

8. Lam KY, Chan AC, Wu PC, Chau KY, Tideman H, Wei W. Desmoplastic variant of ameloblastoma in Chinese patients. $\mathrm{Br} J$ Oral Maxillofac Surg 1998;36, 129-134.

9. Yazdi I, Seyedmajidi M, Foroughi R. Desmoplastic ameloblastoma (a hybrid variant): report of a case and re-view of the literature. Arch Iran Med 2009;12:304-308.

10. Mintz S, Velez I. Desmoplastic variant of ameloblastoma. J Am Dent Assoc 2002;133:1072-1075. 